\title{
Behavioral and neural correlates of memory selection and interference resolution during a digit working memory task
}

\author{
YuJI YI \\ Stony Brook University, Stony Brook, New York \\ NAOMI DRIESEN \\ Yale University School of Medicine, New Haven, Connecticut \\ AND \\ HoI-Chung Leung \\ Stony Brook University, Stony Brook, New York
}

\begin{abstract}
Neuroimaging studies have shown the involvement of prefrontal and posterior parietal cortexes in regulating information processing. We conducted behavioral and fMRI experiments to investigate the relationship between memory selection and proactive interference (PI), using a delayed recognition task with a selection cue presented during the delay indicating which two of the four studied digits were relevant to the present test. PI was indexed by the response time differences between rejecting probes matching and not matching the no longer relevant digits. By varying the delay intervals, we found that the effect of PI did not diminish, even for cases in which the postcue interval was extended to $9 \mathrm{sec}$, but was stronger when the precue interval was lengthened to $5 \mathrm{sec}$. By examining the correlation between PI index and neural correlates of memory selection, we found that stronger PI is predicted by lower selection-related activity in the left inferior parietal lobe, the precuneus, and the dorsal middle frontal gyrus. Our results suggest that activity in the prefrontal-parietal network may contribute to one's ability to focus on the task-relevant information and may proactively reduce PI in working memory.
\end{abstract}

The term working memory refers to the temporary maintenance and processing of information to fulfill ongoing task demands required by complex cognitive tasks such as mental arithmetic and reasoning (Baddeley, 1986; Baddeley \& Hitch, 1974). It is known that the capacity of working memory is rather limited (Cowan, 2001) and that a main source of such limitation is caused by proactive interference (PI), interference from information that has become no longer relevant (Brown, 1954; Underwood, 1957). A dominant view of interference resolution is that attentional processes are involved in selecting taskrelevant information and ignoring irrelevant or no longer relevant information (see reviews by Jonides et al., 2008; Postle, 2006). Such selection processes presumably are mediated by the lateral prefrontal cortex (PFC) and the posterior parietal cortex (PPC) (see Deco \& Rolls, 2005; Desimone \& Duncan, 1995; Miller \& Cohen, 2001).

Behavioral studies have provided evidence to support the involvement of attentional processes in controlling information accessibility in working memory. Cowan (1988, 1995,1999 ) has proposed a component called the focus of attention as a mechanism to keep a small amount of information more accessible. Oberauer (2001, 2002, 2005) refined the model by conducting a set of elegant experiments to test whether information can be removed from the focus of attention in achieving the task goal. In his 2001 study, Oberauer used a modified delayed recognition task; two lists of words were presented as memory sets followed by a cue informing the subjects which list would remain relevant for the later recognition test. Besides finding the typical PI effect or intrusion cost (i.e., longer response times [RTs] to no longer relevant words relative to words not in the original memory sets), he found increases in RT only with an increasing number of relevant words. The number of no longer relevant words did not affect RT when the delay between cue and probe was at least $1 \mathrm{sec}$ (Oberauer, 2001). These findings suggested that, although the relevant words are maintained in the focus of attention for matching with the probe, the no longer relevant words are removed from the focus of attention $1 \mathrm{sec}$ after the cue presentation and no longer occupy the central capacity of working memory. Further, Nobre and colleagues (Lepsien, Griffin, Devlin, \& Nobre, 2005; Lepsien \& Nobre, 2007 ) investigated the effect of memory selection on later probe recognition, using informative cues in comparison with neutral or uninformative cues during visual work- 
ing memory tasks; they observed relatively shorter RTs to cued probes, suggesting that informative cues presented during the delay period can facilitate target recognition (Griffin \& Nobre, 2003; Lepsien et al., 2005). However, it is unclear from these findings whether the process of memory selection has any effect on rejecting familiar nontargets or reducing PI.

Activity in the left ventrolateral PFC has been implicated as the neural correlate of interference resolution (see the review by Jonides \& Nee, 2006). This hypothesis is based on the consistent finding of increases in activity in the left inferior frontal gyrus (IFG) during rejection of negative letter probes of higher as opposed to lower familiarity in neuroimaging studies (D'Esposito, Postle, Jonides, \& Smith, 1999; Jonides, Smith, Marshuetz, Koeppe, \& Reuter-Lorenz, 1998; Nee, Jonides, \& Berman, 2007; Postle, Berger, Goldstein, Curtis, \& D'Esposito, 2001; Zhang, Leung, \& Johnson, 2003). Familiarity in these studies was typically manipulated by varying the recency of a negative probe in the trial history (Monsell, 1978) or by presenting a cue to indicate the relevance of remembered items (Brown, 1954). Converging evidence from neuropsychological studies showed that the ability to inhibit no longer relevant items was disrupted in patients with inferior frontal lobe lesions (Conway \& Fthenaki, 2003; Thompson-Schill et al., 2002). The critical role of the left IFG in controlling PI is further confirmed by the observation of a stronger PI effect (i.e., larger intrusion cost) when repetitive transcranial magnetic stimulation was applied to the left IFG of healthy young subjects, but with no observed effect when the stimulation was applied to control areas such as the primary motor cortex and supplementary motor area (Feredoes, Tononi, \& Postle, 2006). Some authors have further suggested that the left IFG plays a more general role in selecting task-relevant information (Zhang, Feng, Fox, Gao, $\&$ Tan, 2004). However, others have indicated that the left IFG plays a more specific role in resolving PI in the verbal domain, whereas the dorsal PFC and PPC do so in the spatial domain (Leung \& Zhang, 2004; Mecklinger, Weber, Gunter, \& Engle, 2003).

Previous research has associated both the PFC and PPC with selective attention (see Corbetta \& Shulman, 2002; Desimone \& Duncan, 1995), and common activations in these regions have been found during attention and working memory tasks (e.g., Awh \& Jonides, 2001; LaBar, Gitelman, Parrish, \& Mesulam, 1999; Mayer et al., 2007). Some have emphasized the finding of enhanced activity in the lateral PFC, particularly the dorsolateral PFC during maintenance of task-relevant information in the face of interference or distraction (see reviews by Knight, Staines, Swick, \& Chao, 1999; Miller, 2000). Recently, several fMRI studies have examined brain activity during selective encoding and selective maintenance, using a modified delayed recognition task with a cue shown either before or after stimulus presentation, respectively. Nobre and colleagues (Lepsien et al., 2005; Lepsien \& Nobre, 2007; Nobre et al., 2004) found activations in the lateral PFC and PPC, including in the left IFG/anterior insula and precuneus during both selective encoding and selec- tive maintenance of visual information. Nee and Jonides (2009) reported that the left IFG and bilateral intraparietal sulcus are preferentially involved in memory selection, whereas the superior parietal lobe and frontal eye fields are more involved in perceptual selection. These findings together suggest that the subdivisions of the lateral prefrontal and parietal regions may play subtle but different roles in controlling information perception and processing. However, whether the selection-related activity serves as a way to resolve PI at recognition has not been established, inasmuch as previous studies either did not consider PI in the design (Lepsien et al., 2005; Lepsien $\&$ Nobre, 2007; Nobre et al., 2004) or did not fully investigate the relationship between memory selection and PI (Nee \& Jonides, 2009).

The purpose of the present study was to determine the relationship between memory selection and PI resolution in working memory. We implemented the delayed recognition paradigm with a selection cue presented during the delay and manipulated the probe familiarity for indexing PI. We used simpler selection cues (arrows pointing to half of the recently memorized items) as opposed to the more complex cues (words indicating the color or conceptual category of the memorized items) used in previous studies (e.g., Nee \& Jonides, 2009; Oberauer, 2001). In Experiment 1, we examined the effect of postcue delay on PI, which was intended to replicate Oberauer's (2001) findings and to confirm that PI would not diminish with a long delay $(9 \mathrm{sec})$ planned for the fMRI experiment. In Experiment 2, we examined the effect of precue delay on PI, which was to test whether PI would be enhanced by increasing the initial rehearsal time. In Experiment 3, we added a neutral cue condition (as control) to the same behavioral task and acquired fMRI data, which not only allowed us to identify brain regions involved in selective information processing but also allowed us to determine whether postcue brain activity modulates PI at the response stage. If the no longer relevant items were removed from working memory after cue presentation and attention was directed to the relevant items, we would expect to observe activations in the PFC and PPC in correspondence to the selection demand, as in previous studies. It was further expected that, if these regions contribute to successful memory selection, their activity would lead to reduction of PI and facilitation of target recognition.

\section{METHOD}

\section{Subjects}

Twenty adults participated in Experiment 1 (mean age $=20.5$ years, 11 male), 21 participated in Experiment 2 (mean age $=20.5$ years, 7 male), and 18 participated in Experiment 3 (mean age $=26$ years, 7 male). Subjects in the first two experiments were psychology undergraduates in the Stony Brook University campus, and they received course credits for their participation. Subjects in the fMRI experiment were recruited from the Yale-New Haven area, and they were compensated monetarily for their participation. Subjects in all experiments had normal or corrected-to-normal vision. In addition, subjects for the fMRI experiment were screened for major medical problems, neurological or psychiatric disorders, and use of chronic medication. Informed consent was obtained from all the subjects. 


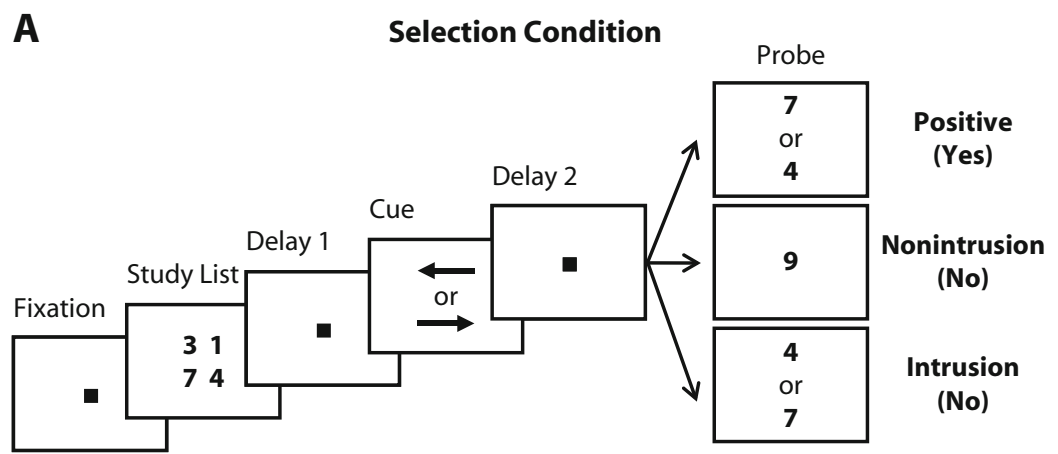

B Neutral Condition

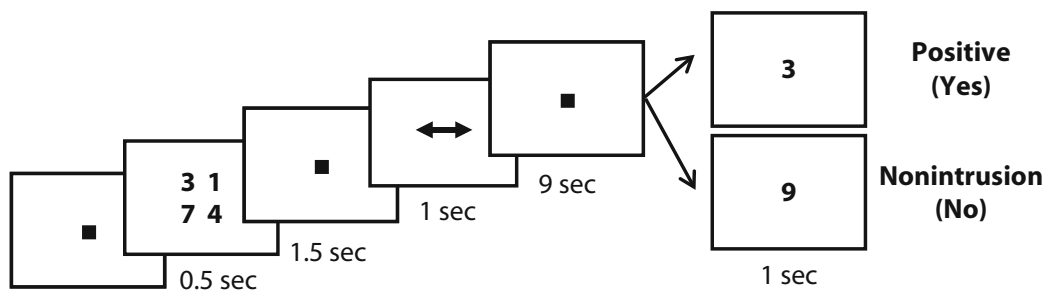

$6 \mathrm{sec}$

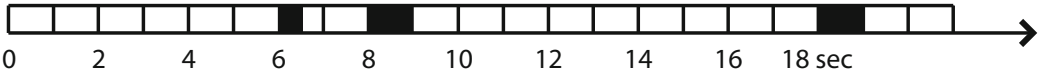

Figure 1. (A) Sequence of trial events during the memory selection condition in all experiments. A memory set of four digits was presented at the beginning of each trial. After a short delay, a selection cue (an arrow pointing either to the left or right) was presented to indicate the two digits to be remembered for the current trial. There were three types of probes: one of the two to-be-remembered digits (positive), a digit not in the original memory set (nonintrusion), or a digit in the original memory set but no longer relevant after the cue presentation (intrusion). Refer to the text for the timing of each event. The durations of Delay 1 and Delay 2 were varied in Experiment 2 and Experiment 1, respectively. (B) Sequence of trial events during the neutral condition in Experiment 3. Different from the selection condition, a neutral cue (double-headed arrow) was presented to indicate that both columns of digits remain relevant for the recognition test. There were two types of probes: one of the four digits in the memory set (positive) or a digit not in the memory set (nonintrusion). The duration of each event is shown underneath each box. The timeline indicates the timing of the events across the entire trial duration, with the shaded portions indicating the study list, cue, and probe presentations, respectively.

\section{Behavioral Experiments: Experiments 1 and 2}

We used a delayed recognition paradigm with a cue presented sometime between the presentation of the study list and the testing probe. In Experiment 1, we varied the gap between the cue and the probe to test whether the effect of PI would diminish over an extended period and to replicate Oberauer's (2001) study in the present experimental context. In Experiment 2, we varied the gap between the study list and the cue to test whether the effect of PI would be enhanced by increasing rehearsal time.

Figure 1A shows the events in a sample trial of our delayed recognition task with a selection cue. At the beginning of each trial, two columns of digits (study list) were presented for $300 \mathrm{msec}$ on the computer screen. The digits were not repeated and were randomly selected between 0 and 9 . After the disappearance of the study list and a short delay (Delay 1), a cue (a left- or right-pointing arrow) was presented for $1 \mathrm{sec}$ in the center of the screen to indicate which column of digits remained relevant (i.e., the final memory set). For example, a left-pointing arrow would indicate that the two digits in the left column should be remembered for the current trial and that those in the right column should be ignored because they were no longer relevant. Following another delay (Delay 2), a probe was pre- sented in the center of the screen. In Experiment 1, Delay 1 was fixed (1 sec), Delay 2 was variable $(100,300,1,000,3,000$, or $9,000 \mathrm{msec})$, and the intertrial interval was $0.5 \mathrm{sec}$. In Experiment 2, Delay 1 was variable $(300,600,1,000,3,000$, or 5,000 msec), Delay 2 was fixed $(2 \mathrm{sec})$, and the intertrial interval was $1 \mathrm{sec}$. There were 10 blocks of trials in each experiment ( 2 blocks per delay condition), and block order was counterbalanced across subjects. The three probe types were mixed in a block. Overall, there were 48 trials for each delay condition ( 24 positive, 12 nonintrusion, and 12 intrusion probes).

The subjects were told to press a key to indicate whether the probe matched one of the digits in the final memory set. The three probe types were positive probe (matches one of the target items), nonintrusion probe (does not match any digits in the original study list), and intrusion probe (matches one of the digits in the study list that has become no longer relevant). Both intrusion and nonintrusion probes were negative probes, and the chance of positive and negative probes was equal. To reduce the effect of trial history, the probe of the current trial did not appear in the previous trial. An error message was presented on the screen if there was an error response. The designation of response (yes/no) and key (left/right) were counterbalanced across subjects. 
Subjects practiced two task blocks before each experiment and completed a postsession interview afterward. The intrusion cost was defined as the RT and accuracy difference between making judgments to the intrusion and nonintrusion probes. RT and accuracy data were analyzed using ANOVAs with probe type and Delay 1 or Delay 2 as within-subjects factors. All paired $t$ tests were corrected for multiple comparisons, and the reported $p$ values included Bonferroni correction. RTs that were at least 3 standard deviations $(S D s)$ away from the mean were removed from analysis $(<2 \%)$.

\section{fMRI Experiment: Experiment 3}

Design and Procedure. The selection condition in Experiment 3 was similar to that in Experiment 1, but a neutral condition was added to serve as a control (Figure 1B). In this condition, a doubleheaded arrow informed the subject that any of the digits initially presented could be targets. The task parameters were identical, as in Experiment 1, except for a longer initial fixation period $(6 \mathrm{sec})$, longer intertrial interval (14 sec), and longer probe presentation $(1 \mathrm{sec})$. Delay $1 \mathrm{was} 1.5 \mathrm{sec}$, and Delay 2 was $9 \mathrm{sec}$. Each trial lasted $33 \mathrm{sec}$. Each subject performed eight runs, with a total of 32 neutral cue trials and 32 selection cue trials. The two cue conditions were presented in random orders and were counterbalanced across runs and subjects.

Image acquisition. Subjects were in a supine position in the scanner. Head movement was restricted with foam pillows and with a band across the forehead. Images were collected with a $3 \mathrm{~T}$ Siemens Trio Scanner using the standard quadrature head coil and $\mathrm{T} 2{ }^{*}$-sensitive gradient-recalled single-shot echo-planar pulse sequence. Twentyfour axial-oblique slices (5-mm thickness, no gap) were prescribed parallel to the anterior-posterior commisural line. The acquisition parameters for the anatomical images were as follows: repetition time $(\mathrm{TR})=300 \mathrm{msec}$, echo time $(\mathrm{TE})=2.5 \mathrm{msec}$, flip angle $=60^{\circ}$, field of view $(\mathrm{FOV})=220 \times 220 \mathrm{~mm}$, matrix dimensions $=256 \times 256$, and 176 volumes/run. The acquisition parameters for the functional images were as follows: $\mathrm{TR}=1.5 \mathrm{sec}, \mathrm{TE}=30 \mathrm{msec}$, flip angle $=$ $80^{\circ}, \mathrm{FOV}=220 \times 220 \mathrm{~mm}$, and matrix dimensions $=64 \times 64$. The voxel resolution was $3.4 \times 3.4 \times 5 \mathrm{~mm}$. Slices within one volume were collected in interleaved fashion. The acquisition parameters for the high-resolution structural images were as follows: $\mathrm{TR}=2.53 \mathrm{sec}$, $\mathrm{TE}=3.52 \mathrm{msec}$, flip angle $=7^{\circ}, \mathrm{FOV}=256 \times 256 \mathrm{~mm}$, matrix dimensions $=256 \times 256$, and slice thickness $=1 \mathrm{~mm}$.

Visual stimuli were projected onto a screen positioned in front of the scanner opening and were viewed through a mirror mounted on the head coil. Visual stimuli were presented in black against a white background and were delivered using Inquisit 1.33 (Millisecond Software, Seattle, WA; www.millisecond.com). The subjects used their index and middle fingers to respond via a two-key button box.

Image processing and analysis. Image processing and analysis were performed using SPM2 (www.fil.ion.ucl.ac.uk/spm/). All image data sets were processed in the following manner. Images were adjusted to approximately the same orientation. Differences in timing of slice acquisition were corrected using linear interpolation. The functional images were corrected for motion: Runs with images of motion greater than $3 \mathrm{~mm}$ in translational or $1.5^{\circ}$ in rotational dimensions were removed. Functional images were then coregistered with the anatomical images. The anatomical images were segmented into gray and white matter and spatially normalized to the Montreal Neurological Institute (MNI) gray-matter template. The normalization parameters were applied to the functional images. The normalized images were smoothed using a Gaussian filter with a full width of $8 \mathrm{~mm}$ at half maximum.

Individual data were analyzed by constructing a general linear model in SPM2. Two regressors were defined for the two events of each task condition: cue ( $8-9 \mathrm{sec})$ and probe $(18-20 \mathrm{sec})$ (timing of each event is from the beginning of a trial). The regressors were convolved with the canonical hemodynamic response function. Motion parameters were incorporated as covariates to remove effects from head motion. Since the average accuracies were high for all conditions, all trials were included in the individual SPM. Individual $t$ maps were generated to reveal activations in correspondence to the cue and probe by contrasting the selection versus the neutral condition. We applied random effects analysis to derive the group composite maps by incorporating the individual data from the firstlevel analysis. The $t$ values for each voxel in the selection-neutral contrasts were calculated using a one-sample $t$ test for the group $(d f=13)$. For visualization, resulting activations were overlaid on the averaged normalized anatomical image of the whole group.

Furthermore, we analyzed the image data to examine the correlation between cue-related activations and individual differences in PI index (intrusion probe RT - nonintrusion probe RT in the selection condition). Using the beta values from the selection-neutral contrast at the cue stage and PI index from each subject, we performed a simple regression. Suprathreshold clusters from the correlation analysis $(p<.05$, FDR corrected) were plotted as regions of interest (ROIs) for visualization. ROIs were spheres with a radius of $8 \mathrm{~mm}$ centered at the peak coordinate of the clusters. Two additional ROIs were defined in the left IFG on the basis of previous reports of their role in interference resolution during working memory tasks (Jonides et al., 1998; Nee \& Jonides, 2009).

\section{RESULTS}

\section{Experiment 1 Data}

The purpose of Experiment 1 was to replicate Oberauer's (2001) findings of intrusion cost, with a longer postcue delay, with digits as stimuli, and with a smaller memory set size (to ensure high performance). Figure 2 shows the RT and accuracy results from Experiment 1. RT data showed significant main effects of probe type $[F(2,38)=15.529, p<.001]$ and Delay $2[F(4,76)=$ $15.758, p<.001]$ but not their interaction $[F(8,152)=$ $1.363, p>.1]$ (see Figure 2A). Accuracy data showed that the main effect of probe type was significant $[F(2,38)=$ $5.017, p<.05]$ and that the interaction between probe type and Delay 2 approached significance $[F(8,152)=$ $1.884, p=.066]$. The average response to the intrusion probe was longer and less accurate than it was to the nonintrusion probe (all $p \mathrm{~s}<.01$ ). However, the manipulation of Delay 2 did not have a significant effect on the intrusion cost [RT, $F(4,76)=1.426, p>.1$; accuracy, $F(4,76)=1.775, p>.1]$ (see Figures $2 \mathrm{C}$ and 2D). On average, the intrusion cost in RT was $63 \mathrm{msec}$ across the postcue delay intervals, which is consistent with previous findings (Oberauer, 2001).

In this experiment, we have extended previous work by showing that the PI effect does not diminish up to $9 \mathrm{sec}$ after the cue presentation. These findings are consistent with the notion that the no longer relevant information, although removed from the focus of attention in working memory, remains highly familiar and influences delayed recognition. In Experiment 2, we varied the delay between the study list and the cue to further test whether the effect of PI would be enhanced by increasing the familiarity level of the no longer relevant digits.

\section{Experiment 2 Data}

The final analysis included data from 20 subjects. Data from 1 subject were excluded from analysis because of psychiatric medication. Figure 3 shows the results from Experiment 2. RT data showed significant effects of probe type $[F(2,38)=15.285, p<.001]$, Delay $1[F(4,76)=$ 
A

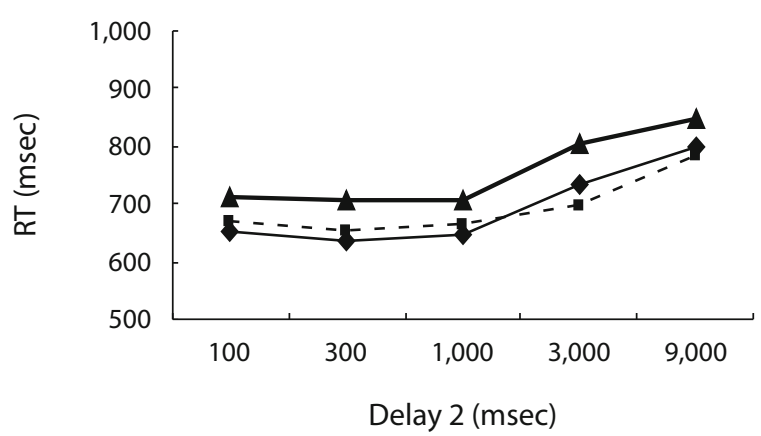

C

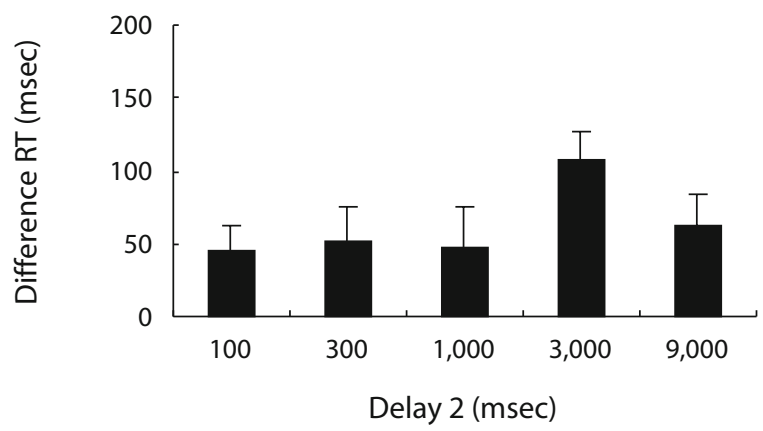

B

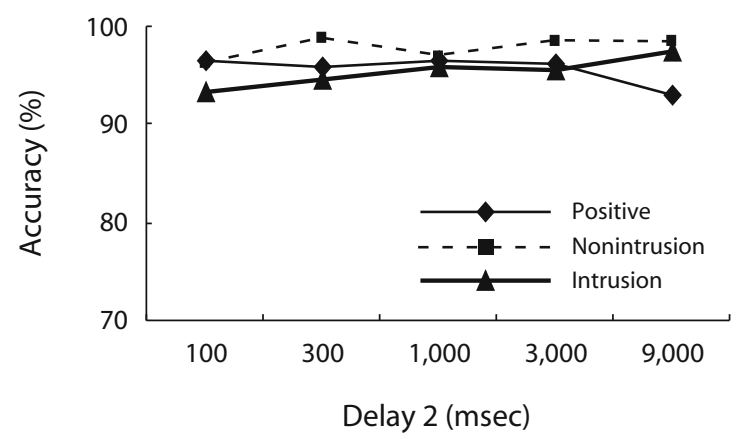

D

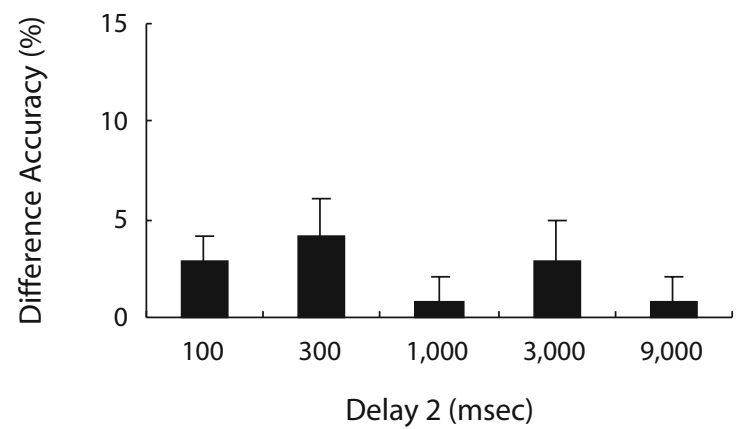

Figure 2. Results from Experiment 1. Effects of postcue delay: (A) Response time (RT) averages for the three types of probes for the five postcue delay (Delay 2) durations. (B) Accuracy averages for the three probes for the five postcue delays. (C) Differences in RT between the intrusion probe and nonintrusion probe at the various delays. (D) Differences in accuracy between the intrusion probe and nonintrusion probe at the various delays. All the values are absolute values.

$6.493, p<.001]$, and their interaction $[F(8,152)=3.338$, $p<.01]$ (see Figure 3A). Accuracy data showed that the main effect of probe type $[F(2,38)=16.672, p<.001]$ and the interaction between probe type and Delay 1 $[F(8,152)=2.584, p<.05]$ were significant (Figure 3B). The average response to the intrusion probe was longer and less accurate compared with that of the nonintrusion probe (all $p \mathrm{~s}<.05$ ). In particular, the manipulation of Delay 1 had a significant effect on intrusion cost in RT $[F(4,76)=3.151, p<.05]$ but not on accuracy $[F(4,76)=$ $1.091, p>.1]$ (Figure 3C and 3D).

Consistent with Experiment 1, the responses to the intrusion probes were longer and less accurate than responses to the nonintrusion probes. Increasing the delay between the study list and cue presentation apparently allowed more rehearsal time and increased the familiarity of the digits. These findings indicate that it is more difficult to disregard well-rehearsed items, thus causing a larger PI effect in the longer delay conditions. In Experiment 3, we examined the neural correlates of memory selection and tested whether activity in the PFC and PPC is involved in resolving PI by focusing on the task-relevant information after the cue.

\section{Experiment 3 fMRI Data}

Data from 14 subjects were included in the final analysis for the present report. Data from 4 subjects were excluded from analysis: 2 for excessive head motion, 1 for technical error, and 1 because his RT averages were $2.5 S D$ greater than the group average. In the remaining sample, the average RT to the intrusion probe $(965 \mathrm{msec})$ was numerically longer than to the nonintrusion $(904 \mathrm{msec})$ and positive (902 $\mathrm{msec}$ ) probes in the selection condition. A one-way ANOVA showed that the differences between the probes in the selection condition approached significance $[F(2,26)=$ $2.928, p=.071]$. In addition, the average $\mathrm{RT}$ to the positive probe in the selection condition was significantly faster than that in the neutral condition $[t(13)=3.189, p<.01]$, whereas the difference between the nonintrusion probes of the two conditions was not significant $[t(13)<1]$. The reduction in $\mathrm{RT}$ to the positive probe in the selection condition compared with the neutral condition is consistent with previous findings in suggesting the facilitative effect of informative selection cues (e.g., Lepsien et al., 2005).

Brain activations in response to the task cues. The main purpose of the fMRI experiment was to test whether the left IFG and posterior parietal regions are involved in selective processing of information in working memory. Overall, similar activation patterns were observed for the selection and neutral conditions. Direct contrast of the selection condition and the neutral condition revealed no activations at a more stringent threshold (FDR corrected, $p<.05$ ), although greater activations were found in the left middle temporal gyrus (MTG), superior temporal 
A
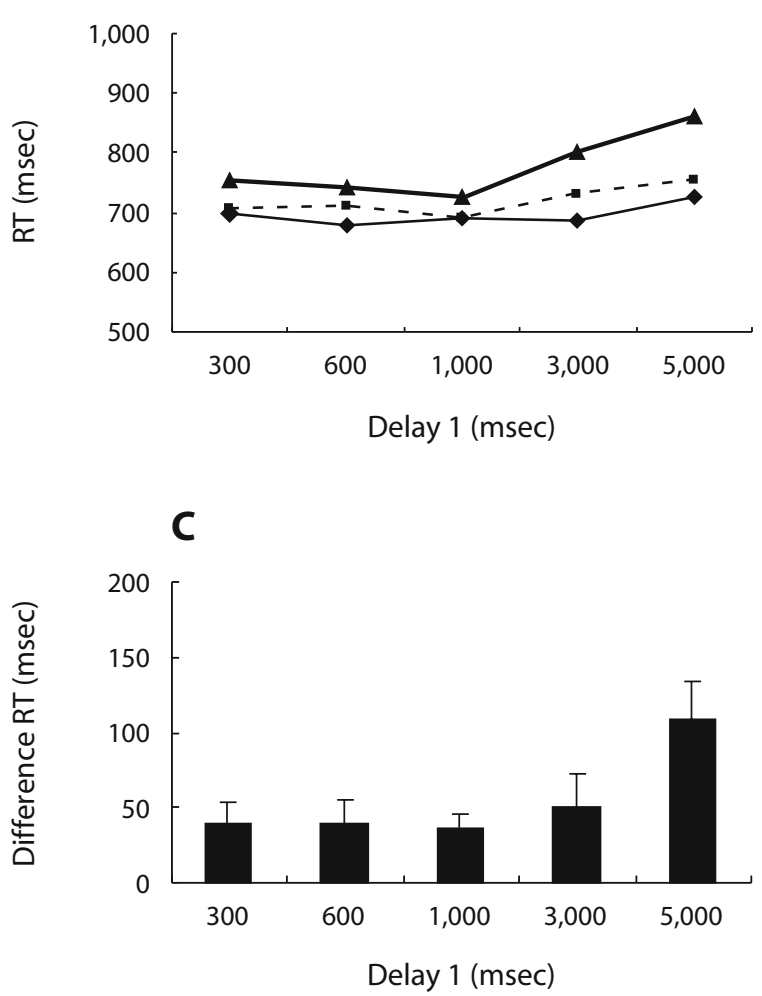

B

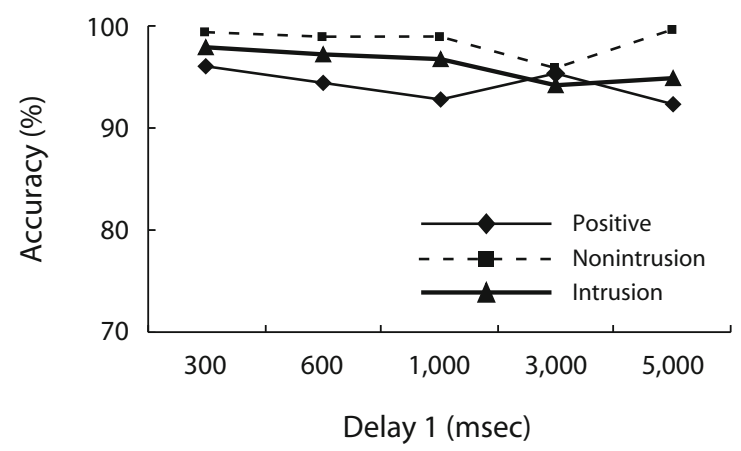

D

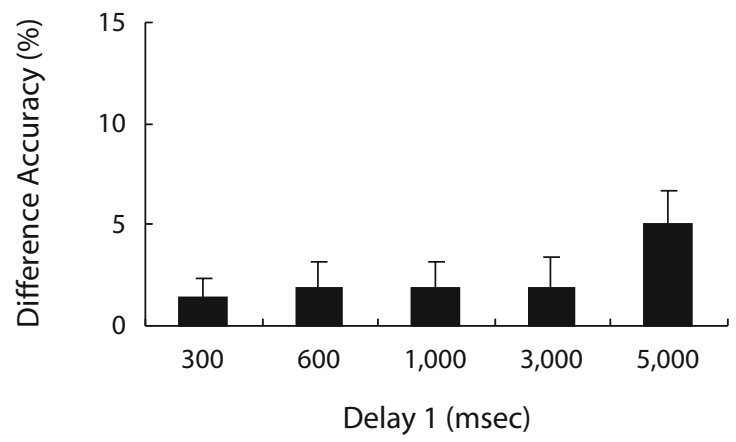

Figure 3. Results from Experiment 2. Effects of precue delay: (A) Response time (RT) averages for the three probe types for the five precue delay (Delay 1) durations. (B) Accuracy averages for the three probes for the five precue delays. (C) Differences in RT between the intrusion probe and nonintrusion probe at the various delays. (D) Differences in accuracy between the intrusion probe and nonintrusion probe at the various delays. All the values are absolute values.

gyrus (STG), cerebellum, and precuneus comparing the selection condition with the neutral condition at a lower threshold ( $p<.001$, uncorrected; cluster size $\geq 9$ voxels) (Figure 4, Table 1). The activation of STG and MTG was expected, as in previous studies of verbal rehearsal (Buchsbaum, Hickock, \& Humphries, 2001; Buchsbaum, Olsen, Koch, \& Berman, 2005; Postle, Berger, \& D'Esposito, 1999). No activations were found in the left IFG, even when the threshold was lowered to $p<.01$ (uncorrected). No regions showed stronger responses to the neutral condition than to the selection condition at the same threshold ( $p<.001$, uncorrected).

Correlations between brain activation and PI. To further determine whether the left IFG and posterior parietal regions are involved in memory selection and resolving PI, we conducted regression analysis to determine the relationship between cue-related activity and behavioral measures of PI. We found a negative correlation between individual differences in PI index (intrusion cost in RT) and selection cue-related activity (beta weights from the selectionneutral contrast) in the lateral PFC and PPC, including the left inferior parietal lobe (IPL), left dorsal middle frontal gyrus (MFG), and precuneus ( $p<.05$, FDR corrected; see Figure 5 and Table 2). The negative correlation indicated that increases in differences in activity between the selection and neutral condition corresponded to decreases in the intensity of PI (Figure 6). To the contrary, cue-related activity in the left IFG areas, derived from ROIs using coordinates obtained from the literature (Jonides et al., 1998; Nee \& Jonides, 2009), did not show significant correlation with individual differences in PI $\left(r^{2}=.185\right.$ and $.058, p \mathrm{~s}>.1$; see bottom two plots in Figure 6).

For confirmation, we tested the correlation between individual differences in PI and cue-related activity in regions identified from the selection-neutral contrast (Table 1). Activity in the medial parietal clusters correlated negatively with the PI index (all $r^{2} \mathrm{~s}=\sim .5, p \mathrm{~s}<.01$ ). It is worth mentioning that the dorsal portion of the medial PPC was common to both the selection-neutral contrast and regression results (compare Tables 1 and 2 and Figures 4 and 5). The left STG/SMG and left cerebellum also showed negative correlations with PI $\left(r^{2}=\sim .3, p \mathrm{~s}<.05\right)$, whereas the left STG and MTG did not show significant effects $\left(r^{2}<.05 ; p \mathrm{~s}>.1\right)$.

\section{DISCUSSION}

Using a digit working memory task, we measured PI behaviorally and the neural correlates of memory selection, and we examined the relationship between behavioral indexes of PI and regional brain activity. Our behavioral findings extended previous research by showing that no longer 

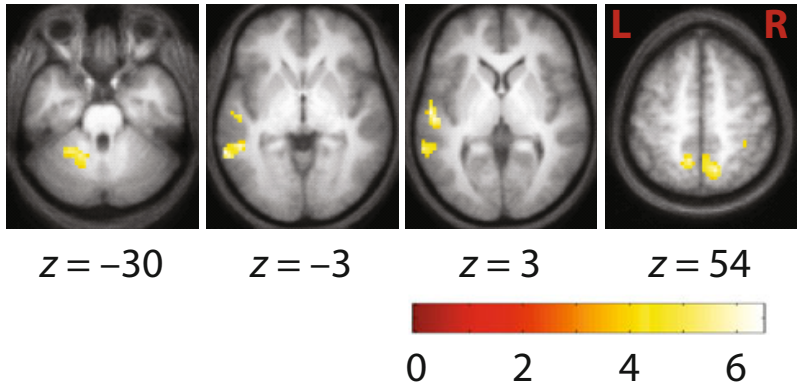

Figure 4. Group activation results from the selection versus neutral contrast at the cue stage $(p<.001$, uncorrected, cluster size $\geq 9$ voxels). See Table 1 for peak $t$ values and coordinates ( $\mathrm{L}=$ left, $\mathrm{R}=$ right $)$.

relevant information can still interfere with recognition response, even $9 \mathrm{sec}$ after the selection cue presentation; also, the interference was stronger when the initial rehearsal time was longer. This suggests that no longer relevant information may remain active for a long time, which may explain the similar level of delay-period activity in the same frontal and parietal regions during the selection condition compared with the neutral condition. Furthermore, our data revealed that the variation in selection-related activity in the dorsal prefrontal and posterior parietal areas is correlated with the PI index. The correlation was negative, suggesting that the prefrontal-parietal network may contribute to one's ability to focus on the task-relevant information in working memory and that the activity in this network may influence later experience of interference caused by the no longer relevant information.

\section{Controlling Relevant and}

\section{No Longer Relevant Information}

On one hand, we showed that the no longer relevant digits can still interfere with delayed recognition $9 \mathrm{sec}$ after the selection cue presentation and that the interference is enhanced with increasing rehearsal time. On the other hand, we found that the selection cue can facilitate behavioral responses to cued digits in the selection condition compared with the noncued digits in the neutral condition. These results are largely consistent with previous studies of PI in verbal working memory (e.g., Oberauer, 2001) and selective attention in visual working memory (e.g., Lepsien et al., 2005). Together, these results suggest that cognitive control of information processing and experience of PI may depend on the efficiency in directing attention to the relevant information (see next section). Such results indicate that removing the no longer relevant information from the focus of attention, according to the Oberauer (2001) findings, can be interpreted as focusing attention to the task-relevant items. It has been proposed that information in the focus of attention is separable from information retrieved from long-term memory (Cowan, 1999) and from information that is no longer needed (Oberauer, 2001, 2002, 2005). The removal of no longer relevant information from the focus of attention may involve active inhibition processes to dampen the goalirrelevant information in memory (Hasher \& Zacks, 1988; Hasher, Zacks, \& May, 1999). We have reported some evidence showing reduced activity in the parahippocampal gyrus and posterior superior parietal lobe (SPL) when pictures of scenes are no longer needed for the later recognition test (Oh \& Leung, in press). However, it is unclear whether that reduced activity was due to active inhibition or to natural decaying. Nevertheless, directing attention to the task-relevant information or inhibition functions does not eliminate PI caused by no longer relevant digits, which most subjects experienced to different extents.

There are at least two explanations for PI. One is that, although the no longer relevant items may be removed from the focus of attention (by directing attention to the task relevant information), their high familiarity can still affect recognition judgment. The result is that it takes longer to reject a familiar item (such as the intrusion probe) than it does a less familiar item (such as the nonintrusion probe). It could also be argued that both the task-relevant and no longer relevant information are equally represented in the working memory system but are distinguished ac-

Table 1

Results From the Selection-Neutral Contrast

\begin{tabular}{|c|c|c|c|c|c|}
\hline \multirow[b]{2}{*}{ Anatomical Structure } & \multirow[b]{2}{*}{ Volume (Voxel) } & \multirow[b]{2}{*}{ Peak $t$ Value } & \multicolumn{3}{|c|}{ MNI Coordinates } \\
\hline & & & $x$ & $y$ & $z$ \\
\hline Left middle temporal gyrus/superior temporal gyrus & 69 & $\begin{array}{l}6.47 \\
5.89\end{array}$ & $\begin{array}{l}-63 \\
-51\end{array}$ & $\begin{array}{l}-48 \\
-45\end{array}$ & $\begin{array}{r}-3 \\
0\end{array}$ \\
\hline Left superior temporal gyrus & 58 & $\begin{array}{l}6.17 \\
4.02\end{array}$ & $\begin{array}{l}-57 \\
-60\end{array}$ & $\begin{array}{r}-15 \\
0\end{array}$ & $\begin{array}{l}3 \\
9\end{array}$ \\
\hline Right medial superior parietal lobe & 104 & $\begin{array}{l}5.86 \\
4.92\end{array}$ & $\begin{array}{r}12 \\
6\end{array}$ & $\begin{array}{l}-63 \\
-51\end{array}$ & $\begin{array}{l}54 \\
57\end{array}$ \\
\hline Left cerebellum & 54 & $\begin{array}{l}5.46 \\
5.27\end{array}$ & $\begin{array}{l}-18 \\
-27\end{array}$ & $\begin{array}{l}-51 \\
-48\end{array}$ & $\begin{array}{l}-30 \\
-30\end{array}$ \\
\hline Left precuneus* & 34 & $\begin{array}{l}5.68 \\
4.16\end{array}$ & $\begin{array}{r}-9 \\
-18\end{array}$ & $\begin{array}{l}-54 \\
-60\end{array}$ & $\begin{array}{l}54 \\
63\end{array}$ \\
\hline Left superior temporal gyrus/supramarginal gyrus* & 30 & 5.66 & -54 & -42 & 24 \\
\hline
\end{tabular}

Note-These regions showed stronger responses to the selection condition than to the neutral condition at the cue stage $(p<.05$, FDR corrected at the cluster level). The $t$ values and Montreal Neurological Institute coordinates (in millimeters) are shown for the peak of the activation clusters. ${ }^{*}$ These clusters approached significance $(p<$ .15 , FDR corrected). 

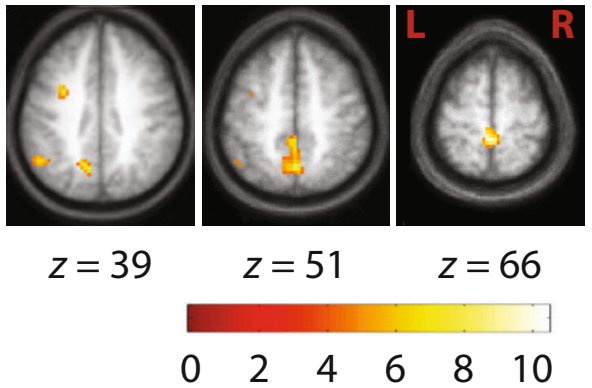

Figure 5. Group activation results from the regression analysis at the cue stage $(p<.05$, FDR corrected, cluster size $\geq 9$ voxels). Clusters show negative correlations between beta weights of the selection-neutral contrast and behavioral index of proactive interference. See Table 2 for peak $t$ values and coordinates $(L=$ left, $\mathbf{R}=$ right).

cordingly at the probe stage, and that prolonged RT to the intrusion probe is due to its complex categorization. Our data support the former argument. If the no longer relevant information is removed from the focus of attention (as has been shown by Oberauer, 2001), responses to the positive probes (matching one of the targets) in the selection condition should be faster than those in the neutral condition. Indeed, the facilitation of recognizing the cued positive probes relative to noncued probes was demonstrated by the behavior data from our fMRI experiment and in a previous study of visual working memory using similar cue manipulations (Lepsien et al., 2005). Moreover, we observed a larger intrusion cost when the rehearsal time was increased to $5 \mathrm{sec}$ in the second behavioral experiment, further supporting familiarity as a main source for PI. Future studies could further manipulate the selection cue to determine the interaction between memory selection and PI.

\section{Prefrontal and Parietal Control of Memory Selection and Proactive Interference}

There are two main observations from our fMRI experiment of memory selection. First, we found that temporal and parietal regions showed greater responses to the selection cue in comparison with the neutral cue. Second, we found that variations in selection-related activity in the left posterior parietal (IPL and precuneous) and dorsal prefrontal (dorsal MFG) areas correlated negatively with individual differences in the behavioral index of PI. Our data suggest the possibility that the prefrontal-parietal network contributes to interference resolution by directing attention to the task-relevant information in working memory.

The involvement of posterior parietal and dorsal prefrontal areas in memory selection corresponds well with recent findings using similar task designs in the verbal domain (Nee \& Jonides, 2009) and in the visual domain (Lepsien et al., 2005; Nobre et al., 2004; Oh \& Leung, in press). Previous studies have consistently found activations in the posterior parietal cortex, including the IPL, SPL, and precuneus, during tasks requiring shifting of spatial attention (Liu, Slotnick, Serences, \& Yantis, 2003; Serences, Schwarzbach, Courtney, Golay, \& Yantis, 2004; Yantis et al., 2002). Similar findings have also been reported for frontal eye fields, and our dorsal MFG activation is close to these previously reported locations (Corbetta et al., 1998; Hopfinger, Buonocore, \& Mangun, 2000; Serences \& Yantis, 2006; Yantis et al., 2002). It is possible that these activations in the dorsal prefrontal and posterior parietal cortexes in the present study reflect shifting attention to the task-relevant information. (It is likely a covert process, since we did not find differences between the left- and right-arrow cues in an exploratory analysis.) Taken together, although very few studies examined the neural correlates of attention orientation in working memory, the findings are pointing to a domain-free system involving the medial posterior SPL, IPL, and dorsal MFG in directing attention to task-relevant information.

In contrast with findings reported by Jonides and colleagues (Jonides et al., 1998; Nee \& Jonides, 2009), the left IFG was not more active in correspondence to the selection cue compared with the neutral cue in our digit working memory task and its activity was not correlated with the PI index. We therefore did not find evidence supporting the left IFG's role in resolving PI. The inconsistent findings are probably due to several differences between

Table 2

Regression Results

\begin{tabular}{lccrrr}
\hline & & \multicolumn{3}{c}{ MNI Coordinates } \\
\cline { 3 - 6 } \multicolumn{1}{c}{ Anatomical Structure } & Volume (Voxel) & Peak $t$ Value & \multicolumn{1}{c}{$x$} & \multicolumn{1}{c}{$y$} & $z$ \\
\hline Left precuneus & 44 & 10.43 & -3 & -39 & 66 \\
& & 6.65 & 0 & -48 & 51 \\
& & 6.52 & -3 & -39 & 51 \\
Left precuneus* & 35 & 8.20 & -15 & -60 & 36 \\
& & 8.15 & 0 & -60 & 51 \\
Left middle frontal gyrus & 45 & 7.67 & -30 & 3 & 39 \\
& & 5.56 & -36 & 0 & 48 \\
Left inferior parietal lobe & 58 & 6.44 & -48 & -57 & 39 \\
\hline
\end{tabular}

Note-These regions showed a negative correlation between beta estimates of the selection-neutral contrast at the cue stage and the behavioral index of PI ( $p<.05$, FDR corrected, cluster size $\geq 9$ ). The $t$ values and Montreal Neurological Institute coordinates (in millimeters) are shown for the peak of the activation clusters. ${ }^{*}$ Cluster separated at a threshold of $p<.02$, FDR corrected. 
Precuneus $(-3,-39,66)$

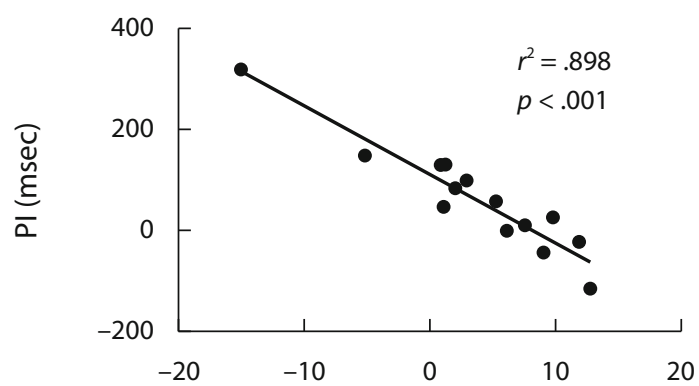

IPL $(-48,-57,39)$

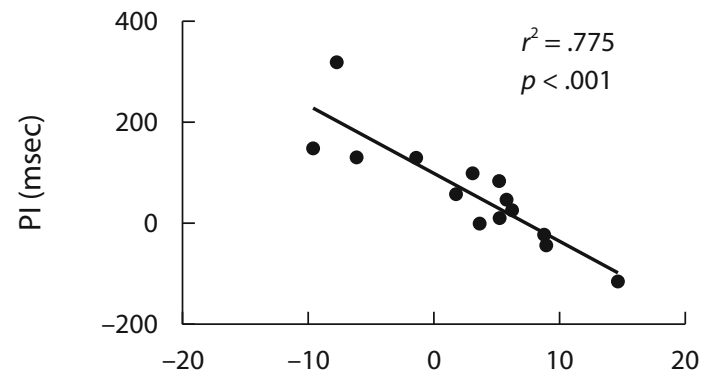

IFG $(-48,21,9)$

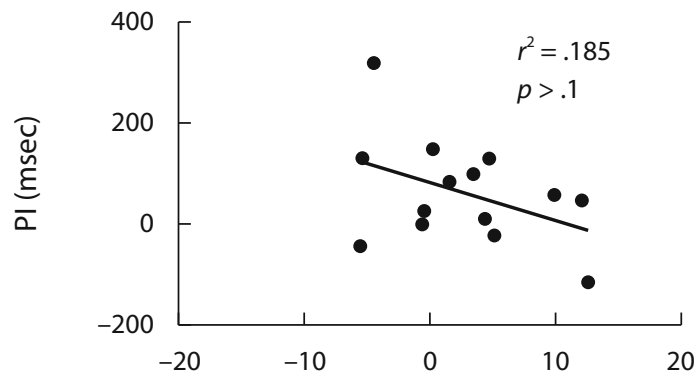

Selection-Neutral Contrast (Beta Weights)
Precuneus $(-15,-60,36)$

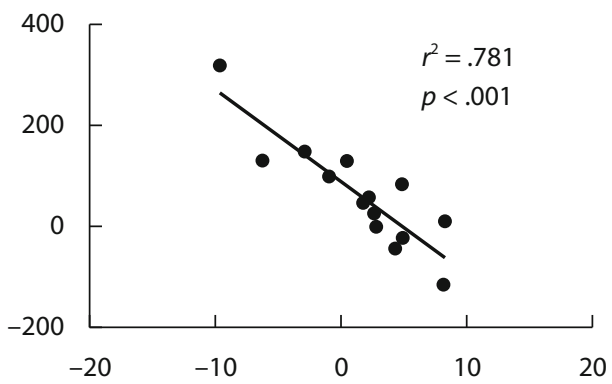

MFG $(-30,3,39)$

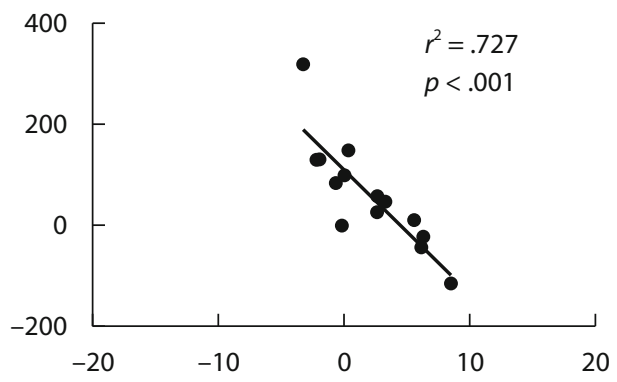

IFG $(-52,22,18)$

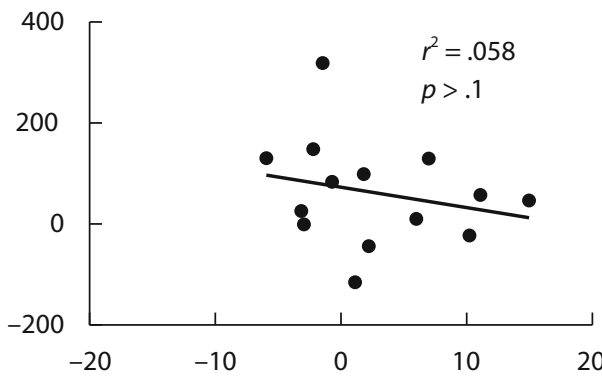

Selection-Neutral Contrast (Beta Weights)

Figure 6. Correlations between selection cue-related activity and behavioral index of proactive interference (PI) are shown for selected regions of interest (ROIs). The top four plots show negative correlations between the beta weights from the selection-neutral contrast and individual differences in PI index (clusters were significant at $p<$ .05 , FDR corrected). Two inferior frontal gyrus (IFG) ROIs (using coordinates from Jonides, Smith, Marshuetz, Koeppe, \& Reuter-Lorenz, 1998, and Nee \& Jonides, 2009) did not show significant correlations between brain activity and PI index. IPL, inferior parietal lobe; MFG, middle frontal gyrus.

the task designs. One major difference is that the two cue conditions in the present study had the same initial memory load whereas the memory selection condition in the Nee and Jonides study had larger initial memory load than their control condition (rehearsal). It is possible that the activations they observed in the left IFG for memory selection may reflect both memory load and selection effects. It is also possible that the same type of competition from distractors occurred during the response stage also occurred during their selection stage under high memory load conditions, as suggested by the investigators to ex- plain their finding of a positive correlation between left IFG activity and PI index (Nee \& Jonides, 2009). Another major difference is that the task used in the present study was much easier than their task and the digits in the present study were presented in two columns. It is possible that in our study, the subjects can easily implement a perceptual selection strategy to direct their attention to the relevant digits. Perhaps that explains the involvement of medial SPL and dorsal MFG in our study, since they were active in the perceptual selection condition in the Nee and Jonides study. Alternatively, the failure to find a signifi- 
cant correlation between left IFG activation and PI index could be a result of the lack of statistical power in our study. This is less likely to be the explanation. We found a weak, insignificant negative correlation in favor of a null effect, whereas Nee and Jonides found a significant positive correlation (i.e., greater IFG activation was associated with higher individual PI index). Further studies would be required to elucidate the role of the left IFG in memory versus perceptual selection and PI resolution.

A potential limitation of this study is that memory load may have confounded the fMRI results. The task-required memory load was four digits in the neutral condition but was two digits in the selection condition, so we may have underestimated the activations in association with memory selection if the same region is sensitive to both memory load and selection. It is also possible that the no longer needed information is still maintained in the same working memory network, as discussed above. When contrasting the delay-period activity in the neutral and selection conditions, no significant differences were found in this comparison. Similarly, load effects (neutral $>$ selection) were not observed in previous studies of visual working memory using similar designs (Lepsien et al., 2005; Oh $\&$ Leung, in press). Since the focus of the present study was to compare memory selection and neutral conditions at the cue stage, we also did not have enough statistical power to measure PI-related activations at the probe stage because of the small number of intrusion and nonintrusion trials per subject.

\section{CONCLUSION}

We observed consistent interference effects in both behavioral experiments, showing that PI is not diminished by extending the postcue delay but is enhanced by extending the precue rehearsal time. The differences in activation in the left IPL, precuneus, and dorsal MFG in response to the selection cue and neutral cue were negatively correlated with individual differences in behavioral index of PI. These findings showed for the first time that activity in the PFC and PPC may proactively reduce PI. Our results thus suggest that selecting or disregarding information in working memory may involve active attentional processes and that a lateral prefrontal-parietal network may be responsible for selecting task-relevant information in working memory and contribute to later interference resolution.

\section{AUTHOR NOTE}

The present work was supported by the State University of New York at Stony Brook, a Drescher Award, and Grant NIH DA024228. We thank Jamie Ferri and Julie Holub for assistance in data collection. We also thank the three anonymous reviewers for their constructive suggestions and comments that greatly improved the manuscript. Address correspondence to H.-C. Leung, Department of Psychology, Stony Brook University, Stony Brook, NY 11794-2500 (e-mail: hoi-chung.leung@sunysb.edu).

\section{REFERENCES}

Awh, E., \& Jonides, J. (2001). Overlapping mechanisms of attention and spatial working memory. Trends in Cognitive Sciences, 5, 119-126.

Baddeley, A. D. (1986). Working memory. New York: Oxford University Press.
Baddeley, A. D., \& Hitch, G. J. (1974). Working memory. In G. H. Bower (Ed.), The psychology of learning and motivation (Vol. 8, pp. 47-89). New York: Academic Press.

BRown, J. (1954). The nature of set-to-learn and of intra-material interference in immediate memory. Quarterly Journal of Experimental Psychology, 6, 141-148.

Buchsbaum, B. R., Hickock, G., \& Humphries, C. (2001). Role of left posterior superior temporal gyrus in phonological processing for speech perception and production. Cognitive Science, 25, 663-678.

Buchsbaum, B. R., Olsen, R. K., Кoch, P., \& Berman, K. F. (2005). Human dorsal and ventral auditory streams subserve rehearsal-based and echoic processes during verbal working memory. Neuron, 48, 687-697.

Conway, M. A., \& FThenaki, A. (2003). Disruption of inhibitory control of memory following lesions to the frontal and temporal lobes. Cortex, 39, 667-686.

Corbetta, M., Akbudak, E., Conturo, T. E., Snyder, A. Z., Ollinger, J. M., DruRY, H. A., ET AL. (1998). A common network of functional areas for attention and eye movements. Neuron, 21, 761-773.

Corbetta, M., \& Shulman, G. L. (2002). Control of goal-directed and stimulus-driven attention in the brain. Nature Reviews Neuroscience, 3, 201-215.

CowAn, N. (1988). Evolving conceptions of memory storage, selective attention, and their mutual constraints within the human informationprocessing system. Psychological Bulletin, 104, 163-191.

Cowan, N. (1995). Attention and memory: An integrated framework. New York: Oxford University Press.

CowAn, N. (1999). An embedded-processes model of working memory. In A. Miyake \& P. Shah (Eds.), Models of working memory: Mechanisms of active maintenance and executive control (pp. 62-101). Cambridge: Cambridge University Press.

CowAn, N. (2001). The magical number 4 in short-term memory: A reconsideration of mental storage capacity. Behavioral \& Brain Sciences, 24, 87-114. doi:10.1017/S0140525X01003922

Deco, G., \& Rolls, E. T. (2005). Attention, short-term memory, and action selection: A unifying theory. Progress in Neurobiology, 76, 236-256.

Desimone, R., \& Duncan, J. (1995). Neural mechanisms of selective visual attention. Annual Review of Neuroscience, 18, 193-222.

D’Esposito, M., Postle, B. R., Jonides, J., \& Smith, E. E. (1999). The neural substrate and temporal dynamics of interference effects in working memory as revealed by event-related functional MRI. Proceedings of the National Academy of Sciences, 96, 7514-7519.

Feredoes, E., Tononi, G., \& Postle, B. R. (2006). Direct evidence for a prefrontal contribution to the control of proactive interference in verbal working memory. Proceedings of the National Academy of Sciences, 103, 19530-19534.

Griffin, I. C., \& Nobre, A. C. (2003). Orienting attention to locations in internal representations. Journal of Cognitive Neuroscience, $\mathbf{1 5}$, 1176-1194.

HASHER, L., \& ZACKS, R. T. (1988). Working memory, comprehension, and aging: A review and a new view. In G. H. Bower (Ed.), The psychology of learning and motivation: Advances in research and theory (Vol. 22, pp. 193-225). New York: Academic Press.

Hasher, L., ZACKs, R. T., \& MAY, C. P. (1999). Inhibitory control, circadian arousal, and age. In D. Gopher \& A. Koriat (Eds.), Attention and performance XVII: Cognitive regulation of performance: Interaction of theory and application (pp. 653-675). Cambridge, MA: MIT Press.

Hopfinger, J. B., Buonocore, M. H., \& Mangun, G. R. (2000). The neural mechanisms of top-down attentional control. Nature Neuroscience, 3, 284-291.

Jonides, J., Lewis, R. L., Nee, D. E., Lustig, C. A., Berman, M. G., \& Moore, K. S. (2008). The mind and brain of short-term memory. Annual Review of Psychology, 59, 193-224.

Jonides, J., \& NeE, D. E. (2006). Brain mechanisms of proactive interference in working memory. Neuroscience, 139, 181-193.

Jonides, J., Smith, E. E., Marshuetz, C., Koeppe, R. A., \& ReuterLORENZ, P. A. (1998). Inhibition in verbal working memory revealed by brain activation. Proceedings of the National Academy of Sciences, 95, 8410-8413.

Knight, R. T., Staines, W. R., Swick, D., \& Chao, L. L. (1999). Prefrontal cortex regulates inhibition and excitation in distributed neural networks. Acta Psychologica, 101, 159-178. 
LaBar, K. S., Gitelman, D. R., Parrish, T. B., \& Mesulam, M. M. (1999). Neuroanatomic overlap of working memory and spatial attention networks: A functional MRI comparison within subjects. NeuroImage, 10, 695-704.

Lepsien, J., Griffin, I. C., Devlin, J. T., \& Nobre, A. C. (2005). Directing spatial attention in mental representations: Interactions between attentional orienting and working-memory load. NeuroImage, 26, 733-743.

Lepsien, J., \& Nobre, A. C. (2007). Attentional modulation of object representations in working memory. Cerebral Cortex, 17, 2072-2083.

LEUNG, H.-C., \& ZHANG, J. X. (2004). Interference resolution in spatial working memory. NeuroImage, 23, 1013-1019.

LiU, T., Slotnick, S. D., Serences, J. T., \& Yantis, S. (2003). Cortical mechanisms of feature-based attentional control. Cerebral Cortex, 13, 1334-1343.

Mayer, J. S., Bittner, R. A., Nikolic, D., Bledowski, C., Goebel, R., \& Linden, D. E. J. (2007). Common neural substrates for visual working memory and attention. NeuroImage, 36, 441-453.

Mecklinger, A., Weber, K., Gunter, T. C., \& Engle, R. W. (2003). Dissociable brain mechanisms for inhibitory control: Effects of interference content and working memory capacity. Cognitive Brain Research, 18, 26-38.

Miller, E. K. (2000). The prefrontal cortex and cognitive control. $\mathrm{Na}$ ture Reviews Neuroscience, 1, 59-65.

Miller, E. K., \& CoHEN, J. D. (2001). An integrative theory of prefrontal cortex function. Annual Review of Neuroscience, 24, 167-202.

Monsell, S. (1978). Recency, immediate recognition memory, and reaction-time. Cognitive Psychology, 10, 465-501.

NeE, D. E., \& Jonides, J. (2009). Common and distinct neural correlates of perceptual and memorial selection. Neurolmage, 45, 963-975.

Nee, D. E., Jonides, J., \& Berman, M. G. (2007). Neural mechanisms of proactive interference-resolution. NeuroImage, 38, 740-751.

Nobre, A. C., Coull, J. T., Maquet, P., Frith, C. D., VandenBerghe, R., \& Mesulam, M. M. (2004). Orienting attention to locations in perceptual versus mental representations. Journal of Cognitive Neuroscience, 16, 363-373.

Oberauer, K. (2001). Removing irrelevant information from working memory: A cognitive aging study with the modified Sternberg task. Journal of Experimental Psychology: Learning, Memory, \& Cognition, 27, 948-957.

Oberauer, K. (2002). Access to information in working memory: Exploring the focus of attention. Journal of Experimental Psychology: Learning, Memory, \& Cognition, 28, 411-421.
Oberauer, K. (2005). Control of the contents of working memory-A comparison of two paradigms and two age groups. Journal of Experimental Psychology: Learning, Memory, \& Cognition, 31, 714-728.

$\mathrm{OH}$, H., \& Leung, H.-C. (in press). Specific and nonspecific neural activity during selective processing of visual representations in working memory. Journal of Cognitive Neuroscience. doi:10.1162/ jocn.2009.21250

Postle, B. R. (2006). Working memory as an emergent property of the mind and brain. Neuroscience, 139, 23-38.

Postle, B. R., Berger, J. S., \& D’Esposito, M. (1999). Functional neuroanatomical double dissociation of mnemonic and executive control processes contributing to working memory performance. Proceedings of the National Academy of Sciences, 96, 12959-12964.

Postle, B. R., Berger, J. S., Goldstein, J. H., Curtis, C. E., \& D'Esposito, M. (2001). Behavioral and neurophysiological correlates of episodic coding, proactive interference, and list length effects in a running span verbal working memory task. Cognitive, Affective, \& Behavioral Neuroscience, 1, 10-21.

Serences, J. T., Schwarzbach, J., Courtney, S. M., Golay, X., \& YANTIS, S. (2004). Control of object-based attention in human cortex. Cerebral Cortex, 14, 1346-1357.

Serences, J. T., \& Yantis, S. (2006). Selective visual attention and perceptual coherence. Trends in Cognitive Sciences, 10, 38-45.

Thompson-Schill, S. L., Jonides, J., Marshuetz, C., Smith, E. E., D'Esposito, M., Kan, I. P., ET AL. (2002). Effects of frontal lobe damage on interference effects in working memory. Cognitive, Affective, \& Behavioral Neuroscience, 2, 109-120.

Underwood, B. J. (1957). Interference and forgetting. Psychological Review, 64, 49-60.

Yantis, S., Schwarzbach, J., Serences, J. T., Carlson, R. L., Steinmetz, M. A., Pekar, J. J., \& Courtney, S. M. (2002). Transient neural activity in human parietal cortex during spatial attention shifts. Nature Neuroscience, 5, 995-1002.

Zhang, J. X., Feng, C.-M., Fox, P. T., GaO, J.-H., \& Tan, L. H. (2004). Is left inferior frontal gyrus a general mechanism for selection? NeuroImage, 23, 596-603.

Zhang, J. X., Leung, H.-C., \& Johnson, M. K. (2003). Frontal activations associated with accessing and evaluating information in working memory: An fMRI study. NeuroImage, 20, 1531-1539.

(Manuscript received September 28, 2008; revision accepted for publication May 31, 2009.) 